\title{
Relations between creaky voice and vocal symptoms of fatigue
}

\author{
Anne-Maria Laukkanen, Leena Rantala \\ Speech and Voice Research Laboratory, Faculty of Social Sciences, \\ Tampere University, Tampere, Finland
}

\begin{abstract}
Background: The use of a creaky voice in speech has become more common. Laryngeal findings relate creaky voice with strong adductive tension in the glottis, and thus it may be expected to be related to an increased risk of vocal fatigue. Methods: The present study investigated the relation of creaky voice use and vocal symptoms in 104 Finnish female university students (mean age 24.3 years, SD 6.3 years). They had no known pathology of voice or hearing. The participants were recorded while reading aloud a text of approximately 40 $\mathrm{s}$ in duration. They also filled in a questionnaire consisting of the Voice Handicap Index (VHI, translation in Finnish), and half of them also answered questions about the frequency and severity of symptoms of vocal fatigue. The samples were perceptually analysed for the amount of creakiness and strain by a speech therapist and a voice trainer. Results: The interrater reliability of the listeners was acceptable (Pearson's $x^{2}=100.159, p=0.000$ for creak; $x^{2}=$ 69.199, $p=0.000$ for strain). Neither creakiness nor strain correlated with vocal symptoms. Participants with a low and a high amount of creakiness or strain did not differ from each other in terms of vocal symptoms or VHI scores. Symptoms' total score correlated with VHI total score and total scores of VHI's physical subscale. Creakiness and strain correlated positively with each other $(r=0.40, p=0.000)$. Conclusion: No significant relations were found between creakiness or strain and vocal symptoms in this sample of university students.
\end{abstract}




\section{Introduction}

Creaky voice, vocal fry or laryngealization are terms that are often used more or less synonymously in voice science [1] although several differences have also been noticed. In general, the expression "vocal fry" is used for a low-pitched sound perceived consisting of a series of taps [2]. Physiologically it has been explained either as a low-pitched modal register phonation [3] or as a register of its own [4-9]. The vocal folds are shortened, thick, strongly adducted, and the false vocal folds are often adducted as well and may be lying on top of the vocal folds. Only a small part of the membraneous vocal folds vibrates with a small amplitude and slow rate, and there are often double pulses visible $[5,9]$. The closed phase of the glottis is long resulting in strong damping of sound energy between successive pulses, hence causing the perception of tapping rather than continuous sound $[3,10]$. The perception of pitch in creaky voice is often unclear, and the voice quality may sound harsh or rough, due to irregular vocal fold vibration. Creaky voice may have a higher perceived pitch compared to vocal fry but with some harsh or dry rasping flavour. Creaky voice has been used as a general term classified into various types of acoustic signals that have been labelled as prototypical creaky voice, vocal fry, multiply pulsed voice, aperiodic voice, tense or pressed voice and non-constricted creak [11]. These different types of signals may or may not be related to very low f0.

All different types of creaky voice may be used as variants of glottalization which refers to the linguistic use of the particular voice quality. Creaky voice may be used to differentiate between words [12], to mark word and phrase boundaries or turn taking in conversation [13-16]. It occurs more typically in low-height than high-height vowels [17]. Creaky voice may also signal hesitancy, complaining or boredom [12, 18] or even suppressed rage [19]. Further, speakers have been found to express their social status - higher education, urban orientation and authority - by using vocal fry [20]. Speakers may also match the amount of vocal fry to that of 
their conversational partner, and this vocal adaptation has been reported to increase satisfaction to the interaction [21].

Vocal fry or creaky voice has been found in both genders. In Britain in the 1990s, the males were reported to use vocal fry from 3 to 10 times more than females, and it was suggested to be more pronounced in cultures where males have special pressures to show masculinity [14]. However, at the moment vocal fry seems to be more typical for females than males, e.g. in the USA $[20,22,23]$. In the study by Gibson [24], $86 \%$ of the female participants used at least one episode of fry in their voice samples. Vocal fry has been detected in the voices of vocally healthy women of different ages, and it may occur in all sentence positions [25].

The amount of creaky voice use in speakers without voice disorders has increased remarkably [20, 26-28]. In Finland, the use of creaky voice has become very common, not only in ordinary speakers, but also in professional TV and radio speakers (editors, announcers, newscasters). Recent studies of Finnish teenagers reported of the large degree of creakiness in both genders [27, 28]. According to Ketolainen et al. [28], 60\% of the 16- to 17 -year-old males and all except one of the females used a lot of creaky voice. In another Finnish study, it was found that some female teachers had vocal fry as much as $16-54 \%$ in their speech [29]. So far, the amount of vocal fry in Finnish adults has not been investigated in a larger number of participants.

According to various authors creaky voice or vocal fry are related to excessive laryngeal tension [30]. Thus, creaky voice may be expected to be related to an increased risk of vocal fatigue. It has also been claimed that people who use frequent creaky voice or vocal fry also complain about vocal fatigue [30, 31] and a lump feeling in the throat [30].

In this retrospective pilot study we investigated (1) the prevalence of creaky voice use and the prevalence of strained voice quality in young Finnish female university students and (2) the relation of the amount of perceived creakiness and strain with the amount of self-reported 
symptoms of vocal fatigue and voice-related handicap. In addition, we explored qualitatively (3) what creaky voice types [11] could be found in the acoustic signals of the voice samples.

\section{Methods}

\section{Recordings}

Originally, 128 female university students without any known pathology of voice or hearing volunteered to participate in a vocal screening as part of their orientation course in their university studies. The participants were recorded while reading aloud a text of 77 words, approximately $40 \mathrm{~s}$ in duration (Finnish version of The Northwind and the Sun) and sustaining a prolonged [a:]. The samples were recorded in a sound-treated studio using a head-set microphone (AKG C44L) placed at $6 \mathrm{~cm}$ distance from the middle of the speaker's lips at a 45 degree angle, and a PC with iFocusrite soundcard. A sampling rate of $44.1 \mathrm{kHz}$ and bit depth 16 were used.

In a preliminary perceptual evaluation, performed by 5 speech therapists, 15 out of 128 participants received grade $\geq 1$ on the GRBAS scale, suggesting some degree of dysphonia. These participants were excluded from the present study. Additionally, 13 other participants were excluded since they had not filled in the self-evaluation questionnaires properly. Finally, the material of the present study included 104 female university students (mean age 24.3 years, SD 6.3 years). The voice quality in the samples of these participants was pre-evaluated by the two judges of the present study and found to be non-dysphonic $(G=0)$. The criteria used for distinguishing dysphonic roughness from creaky voice were that dysphonic roughness occurs more or less constantly in the sample (or occurs very randomly) and involves pronounced irregularity and turbulence noise, while creakiness mainly occurs in the endings of phrases and sentences, has a more regular quality than roughness and typically does not include turbulence noise. 


\section{Self-Evaluation}

All the 104 participants filled in a questionnaire on the Internet consisting of the Voice Handicap Index (VHI, Finnish version that is widely used in clinics in Finland), and 51 participants had also filled in a questionnaire with questions about the frequency and severity of symptoms of vocal fatigue. This asymmetry of the use of the questionnaires is due to the fact that this study was retrospective. The VHI consists of 30 statements in 3 categories (functional, physical and emotional). Each statement is answered on a scale from 0 to $4(0=$ never, $1=$ almost never, 2 = sometimes, 3 = almost always, 4 = always). The total score may thus range from 0 to 120 [32]. Questions about the frequency and severity of symptoms of vocal fatigue (9 symptoms in total) were answered on a scale from 0 to 6 . The scores were added for a sum score that may range from 0 to 108. The questions/statements about the vocal symptoms consisted of the following: (1) "My voice gets tired": frequency of the symptom $(0=$ never, $1=$ sometimes/occasionally, $2=$ sometimes/a couple of times per year, $3=$ more often than $a$ couple of times per year, $4=$ quite often/once $a$ month, $5=$ more often than once a month, $6=$ very often/almost every week), severity of the symptom ( $0=$ no symptom at all, $1=$ some symptom, $2=$ mild symptom, $3=$ more than mild symptom, $4=$ moderate symptom, $5=$ more than moderate symptom, 6 = severe symptom); similarly, (2) "My voice gets hoarse without flu," (3) "I feel a lump or mucus in my throat," (4) "I feel irritation or tickle in my throat," (5) "I feel tiredness or pain in my throat and neck after speaking," (6) "I feel tiredness or pain in my throat and neck after singing," (7) "My voice breaks or fails me when I am speaking," (8) "I lose my voice completely without having a cold," (9) "After a working day my voice is so tired that it has a negative effect on social communication/being with family/participation in vocally demanding activities." The short voice questionnaire is a modification of a questionnaire that has been found to reveal voice disorders effectively [33]. This short questionnaire has been developed since there is a practical need for an easy and quick questionnaire to be used in large screening tests. 


\section{Perceptual Evaluation}

The recorded samples were perceptually analysed by a speech therapist and a voice trainer, both being voice specialists with long experience. The amount of creakiness and that of strain were evaluated separately using a 4 -point scale $(0=$ not at all, $1=$ small amount, $2=$ moderate amount, $3=\mathrm{a}$ lot). The evaluation was based on the subjective impression of how frequently and in how long sections creakiness/strain occurred in the text samples. The samples were listened to in free field from a PC, via a Genelec loudspeaker. Each sample could be listened to as many times as the judges wanted.

\section{Acoustic Analysis}

The samples were qualitatively analysed for the types of creaky voice. This was done by studying the fo curve, oscillogram and fast Fourier transform (FFT) spectra, made by Praat software (version 5.4.05) [34].

\section{Statistical Analyses}

Interrater reliability of the perceptual analysis was studied with cross-tabulation, calculating Pearson's $x^{2}$. Average values (mean, SD, min.- max.) were calculated for the vocal symptoms and results from the VHI. Correlations (Spearman rank correlation coefficient $\rho$ ) were calculated between the mean amount of creakiness and strain and vocal symptoms' total score and VHI total score and subscores. Student's $t$ tests were made to study whether the subjects with a larger degree of creakiness or strain differed from those with a small amount relative to vocal symptoms and VHI score. Statistical analyses were made with SPSS software (IBM SPSS Statistics version 24, Armonk, NY, USA). 


\section{Results}

Reliability of the Perceptual Analysis

Interrater reliability of the two listeners was acceptable (Pearson's $x^{2}=100.159, p=$ 0.000 for creaky voice; $x^{2}=69.199, p=0.000$ for strain). Therefore, mean values for both creakiness and strain were calculated to be used for correlation analyses.

\section{Prevalence of Creakiness, Strain and Vocal Symptoms}

Prevalence of perceived creakiness and strain can be seen in Table 1 . Table 2 shows averages of self-perceived vocal symptom scores. In the perceptual pre-evaluation, all subjects were assessed to have normal, non-dysphonic voice. According to $\mathrm{VHI}$ total scores, the subjects reported on average only a mild vocal handicap. Ten subjects (9.6\%) had VHI total scores between 30 and 58, thus to be classified as having a moderate vocal handicap [32]. Symptoms of vocal fatigue also got low total scores (mean 5.53 and maximum 36 on a scale from 0 to 108).

\section{Relations between Voice Qualities and Vocal Symptoms}

Neither mean creakiness nor mean strain correlated with vocal symptoms (total score of symptoms of vocal fatigue and VHI sum scores; Table 3).

According to $t$ test results, participants with a high or low amount of creakiness or strain did not differ significantly from each other for the mean amount of vocal symptoms, either (Table 4). Creakiness and strain correlated positively with each other (Spearman's $\rho=0.40, p=0.000$; Fig. 1).

\section{Creaky Voice Types}

It was also of interest to study what types of creaky voice [11] were presented in the samples. Creaky voice types were examined by inspecting the fo curves, oscillograms and spectra of the samples. The majority of creakiness in the samples represented either the 
prototypical creaky voice (Fig. 2), generally appearing at phrase endings, or multiply pulsed voice (Fig. 3), which could appear in the beginning of words starting with vowels and continue through many words in the sentences. Figure 2 shows a typical example of the beginning of a prototypical creaky section. A normal non-tense sounding voice quality gradually turns into tense, and finally the creaky voice with a low fo appears.

\section{Discussion}

This study investigated the prevalence of creaky voice in young Finnish female university students and the connection of creakiness with strain and vocal symptoms. According to the results, in total $73 \%$ of the participants were evaluated to use either slight or moderate amounts of creaky voice. This is in line with the results by Wolk et al. [23] who reported that over two thirds of young American females used habitual vocal fry in their speech. In the study by Gottliebson et al. [35], 14\% of the participants (first-year students to become speech language pathologists) showed continuous creaky voice. These participants (who were females) were evaluated as dysphonic. In our study the voices were pre-evaluated as non-dysphonic. The argument for this evaluation (made by the two judges of the present study) is that nowadays the appearance of creaky voice in speech is so common that it cannot be judged as abnormal and a sign of a voice disorder, as long as the voice quality otherwise sounds normal (i.e., creakiness sounds like being a characteristic that is more or less voluntarily used and does not exist in all parts of the sample). In our study, the mean VHI total score value was 15.6 (SD 10) which suggests just a minimal amount of handicap. Ten participants (out of 104, i.e., 9.6\%) had the total score of $\mathrm{VHI}$ between 30 and 58 suggesting moderate handicap. However, creakiness or strain did not correlate with vocal symptoms, and those students with a low (0-1) or a high $(>1<$ 3) mean value of creakiness or strain did not differ from each other in the amount of vocal symptoms and VHI scores. In previous studies [36] the disagreement between voice symptoms 
and perceived voice quality has been a typical observation in participants without diagnosed voice disorder.

The fact that no direct relation was found between creakiness and strain and vocal symptoms may be due to various causes. The amount of vocal symptoms naturally depends on many factors like the amount and type of voice use and the participants' sensitivity to recognize vocal symptoms. It is possible that the participants just do not use their voices so much that they would get symptoms of vocal fatigue. It is also possible that the participants do get vocally overloaded but do not pay attention to the symptoms. Furthermore, there may be individual differences in the vulnerability of the vocal fold tissue to overloading. With a larger sample of participants we might get different results. Additionally, we may always ask whether a text reading sample recorded in a studio corresponds to the person's habitual speech sufficiently. However, according to the perceptual impression of the authors, the participants' text reading samples corresponded well to their spontaneous speech during recording sessions and elsewhere, e.g. during classes. We may, thus, also consider the possibility that the use of creaky voice does not necessarily increase vocal loading and cause an increased risk of vocal fatigue.

It is known that creaky voice appears in different types [11], and for instance it is not always related to a constricted larynx. In creaky voice (vocal fry), subglottal pressure and f0 are typically low, and the amplitude of vocal fold vibration is small $[7,37,38]$. Therefore, the impact stress (pressure per unit area) imposed on the vocal fold tissue during collisions should be low in creaky voice (vocal fry) [39]. Impact stress, in turn, is regarded as the most detrimental type of mechanical stress related to voice production [40]. However, the low subglottal pressure may be more typical in creakiness that appears in phrase and sentence endings where the lung volume is low. In continuous creaky voice in which a higher pitch is heard, subglottal pressure should be higher. In most cases in creaky voice the glottal constriction with strong medialization of the vocal folds seems to be present. In the material of our study, perceptually evaluated 
creakiness and strain also correlated positively $(\rho=0.40, p=0.000)$. This suggests that in this material, the creaky voice use was most likely related to a constricted glottis. Increased medialization of the vocal folds also increases impact stress [39]. Habitual use of strong adduction also loads the adductory muscles and the surface layers of the vocal processes. According to Ylitalo and Hammarberg [41], contact granulomas are typical in speakers with creaky voice. According to clinical observations, habitual creaky voice increases vocal fatigue and may potentially be seen as a type of vocal abuse [30, 31].

It seems plausible to suggest that whether or not creaky voice use increases vocal loading is dependent on the type of creakiness used.

In the present study we did not systematically investigate the amount of different types of creaky voice and their potential relations to the symptoms. Future studies should address this. The results in Venkatraman and Sivasankar [42] showed that at least simulated continuous vocal fry leads to some increase in phonation threshold pressure and perceived effort in a 30 min vocally loading test. The effects of habitual creaky voice use should also be investigated with a vocally loading test. It is also necessary to include laryngeal inspection, although it is expensive and time consuming and thus not often possible to be run for large-scale screening purposes as the present study. Future modelling and high-speed studies are warranted on the measured and estimated mechanical stresses related to different types of creaky voice.

\section{Conclusions}

This retrospective preliminary study investigated the prevalence of habitual creaky voice use in young Finnish females and the relations between creakiness, strain and vocal symptoms. In total, $73 \%$ of the participants were evaluated to use a slight or moderate amount of creaky voice. The prevalence of slight or moderate strain was $88.5 \%$. Creakiness and strain correlated with each other. The amount of creaky voice or strain did not correlate with symptoms of vocal 
fatigue or with answers to the $\mathrm{VHI}$ questionnaire in this material. As self-evaluation includes various shortcomings, future research should include also laryngeal inspection and vocally loading tests. In general, the large prevalence of creaky voice use in young adults warrants an intense research effort in order to increase knowledge whether special pedagogical approaches should be adopted to prevent a potential vast increase in the prevalence of dysphonia in the near future due to excessive habitual creaky voice use.

\section{Acknowledgements}

We thank Ms. Senni Vesterinen for her help in preparing the figures.

\section{Statement of Ethics}

An approval from the Ethical Committee of Tampere University was not necessary to apply for the present study, since the material was collected as a part of the normal teaching procedure, which does not involve any physical or ethical risks for the participants, and whose purpose is to gather information of the participant's voice that will be helpful for the participant him/herself. Material was collected and saved, and the results of the analyses were published respecting the Finnish Data Protection Act (chapter 5, section 31). The participants gave their written informed consent.

\section{Disclosure Statement}

The authors have no commercial or other interests to declare.

\section{Funding Sources}

The study has been carried out funded by Tampere University. 


\section{Author Contributions}

The authors are responsible for collecting and analysing the data and for writing the text.

\section{References}

$1 \quad$ Hollien $\mathrm{H}$. On vocal registers. J Phonetics. 1974;2(2):125-44.

2 Laver J. The phonetic description of voice quality. Cambridge, UK: Cambridge University Press; 1980.

3 Titze IR. Principles of Voice Production. Iowa City: National Center for Voice and Speech; 2000., http://books.google.fi/books?id=ytAeAQAAMAA

4 Moore P, Von Leden H. Dynamic variations of the vibratory pattern in the normal larynx. Folia Phoniatr (Basel). 1958;10(4):205-38.

5 Hollien H, Moore P, Wendahl RW, Michel JF. On the nature of vocal fry. J Speech Hear Res. 1966 Jun;9(2):245-7.

6 Hollien H, Michel JF. Vocal fry as a phonational register. J Speech Hear Res. 1968 Sep;11(3):600-4.

7 Blomgren M, Chen Y, Ng ML, Gilbert HR. Acoustic, aerodynamic, physiologic, and perceptual properties of modal and vocal fry registers. J Acoust Soc Am. 1998 May;103(5 Pt 1):2649-58.

8 Chen Y, Robb MP, Gilbert HR. Electroglottographic evaluation of gender and vowel effects during modal and vocal fry phonation. J Speech Lang Hear Res. 2002 Oct;45(5):821-9.

9 Whitehead RL, Metz DE, Whitehead BH. Vibratory patterns of the vocal folds during pulse register phonation. J Acoust Soc Am. 1984 Apr;75(4):1293-7.

10 Wendahl RW, Moore GP, Hollien H. Comments on vocal fry. Folia Phoniatr (Basel). $1963 ; 15(4): 251-5$. 
11 Keating P, Garellek M, Kreiman J. Acoustic properties of different kinds of creaky voice. Proceedings of the 18th International Congress of Phonetic Sciences, Glasgow, Scotland, 2011.

12 Abercrombie D. Elements in general phonetics. Edinburgh, UK: Edinburgh University Press; 1971.

13 Kreiman J. Perception of sentence and paragraph boundaries in natural conversation. $\underline{\mathrm{J}}$ Phonetics. 1982;10(2):163-75.

14 Henton C, Bladon A. Creak as a socio-phonetic marker. In: Hyman LM, Li CN, editors. Language, Speech and Mind: studies in honor of Victoria A. Fromkin. Beckenham: Croom Helm; 1988. pp. 3-29.

15 Redi L, Shattuck-Hufnagel S. Variation in the realization of glottalization in normal speakers. J Phonetics. 2001;29(4):407-29.

16 Ogden R. Turn-holding, turn-yielding, and laryngeal activity in Finnish talk-in-interaction. $\underline{\mathrm{J}}$ Int Phon Assoc. 2001;31:139-52.

17 Cantor-Cutiva LC, Bottalico P, Ishi CT, Hunter EJ. Vocal fry and vowel height in simulated room acoustics. Folia Phoniatr Logop. 2017;69(3):118-24.

18 Gobl C, NiChasaide A. The role of voice quality in communicating emotion, mood and attitude. Speech Commun. 2003;40(1-2):189-212.

19 Fonagy I. Mimik auf glottaler Ebene. Phonetica. 1962;8(4):209-19.

20 Yuasa IP. 2010; Creaky voice: A new feminine voice quality for young urban-oriented upwardly mobile American women? Am Speech. 2010;85(3):315-37.

21 Borrie SA, Delfino CR. Conversational entrainment of vocal fry in young adult female American English speakers. J Voice. 2017 Jul;31(4):513.e25-32.

22 Abdelli-Beruh NB, Wolk L, Slavin D. Prevalence of vocal fry in young adult male American English speakers. $\underline{\mathrm{J} \text { Voice. }} 2014$ Mar;28(2):185-90. 
23 Wolk L, Abdelli-Beruh NB, Slavin D. Habitual use of vocal fry in young adult female speakers. J Voice. 2012 May;26(3):e111-6.

24 Gibson TA. The role of lexical stress on the use of vocal fry in young adult female speakers. J Voice. 2017 Jan;31(1):62-6.

25 Oliveira G, Davidson A, Holczer R, Kaplan S, Paretzky A. A comparison of the use of glottal fry in the spontaneous speech of young and middle-aged American women. $\underline{\mathrm{J}}$ Voice. 2016 Nov;30(6):684-7.

26 Hornibrook J, Ormond T, Maclagan M. Creaky voice or extreme vocal fry in young women. NZ Med J (Online). 2018 Nov 30;131:36-40.

27 Härkönen R. Situational effects on the acoustic and perceptual characteristics of speech in 14-year-olds [MA thesis]. Tampere, Finland: University of Tampere; 2016. Finnish.

28 Ketolainen I, Laakso M, Simberg S. 16-17-vuotiaiden suomalaisnuorten puheäänen korkeus (Speaking pitch in 16- to 17-year-old Finnish teenagers). Puhe ja kieli (Speech and Language). 2017;37(4):259-277.

29 Pirilä S, Pirilä P, Ansamaa T, Yliherva A, Sonning S, Rantala L. Relationship between Activity Noise, Voice Parameters, and Voice Symptoms among Female Teachers. Folia Phoniatr Logop. 2017;69(3):94-102.

30 Stemple JC, Glaze KB, Klaben BG. Pathologies of the laryngeal mechanism. In: Stemple JC, Roy N, Klaben BK, editors. Clinical voice pathology: theory and management. San Diego: Plural Publishing; 2018. p. 129-30. Electronic version.

31 Colton RH, Casper JK, Leonard RJ. Understanding voice problem: a physiological perspective for diagnosis and treatment. 4th ed. Alphen aan den Rijn: Wolters Kluwer Health Adis (ESP); 2011.

32 Jacobson BH, Johnson A, Grywalski C, Silbergleit A, Jacobson G, Benninger MS, et al. The Voice Handicap Index (VHI): development and validation. Am J Speech Lang Pathol. 1997;6(3):66-70. 
33 Simberg S, Sala E, Laine A, Rönnemaa AM. A fast and easy screening method for voice disorders among teacher students. Logoped Phoniatr Vocol. 2001;26(1):10-6.

34 Boersma P, Weenink D. Praat [computer program]: doing phonetics by computer (version 5.3.57). Amsterdam: Institute of Phonetic Sciences; 2013.

35 Gottliebson RO, Lee L, Weinrich B, Sanders J. Voice problems of future speechlanguage pathologists. J Voice. 2007 Nov;21(6):699-704.

36 Kankare E, Liu D, Laukkanen AM, Geneid A. EGG and acoustic analyses of different voice samples: comparison between perceptual evaluation and voice activity and participation profile. Folia Phoniatr Logop. 2013;65(2):98-104.

37 Murry T, Brown WS Jr. Subglottal air pressure during two types of vocal activity: vocal fry and modal phonation. Folia Phoniatr (Basel). 1971;23(6):440-9.

38 Moore GP. Organic voice disorders. Englewood Cliffs: Prentice-Hall; 1971.

39 Jiang JJ, Titze IR. Measurement of vocal fold intraglottal pressure and impact stress. $\underline{\mathrm{J}}$ Voice. 1994 Jun;8(2):132-44.

40 Titze IR. Mechanical stress in phonation. $\underline{\text { J Voice. }} 1994$ Jun;8(2):99-105.

41 Ylitalo R, Hammarberg B. Voice characteristics, effects of voice therapy, and long-term follow-up of contact granuloma patients. J Voice. 2000 Dec;14(4):557-66.

42 Venkatraman A, Sivasankar MP. Continuous Vocal Fry Simulated in Laboratory Subjects: A Preliminary Report on Voice Production and Listener Ratings. Am J Speech Lang Pathol. 2018 Nov;27(4):1539-45. 
Fig. 1. Correlation between creaky and strained voice qualities $(\rho=0.4, p=0.000)$. Scale $0-3 ; 0$ $=$ no creakiness/strain; 3 = a lot of creakiness/strain. Number of voice samples 104; voice quality assessed perceptually by a speech therapist and a voice trainer.

Fig. 2. a Prototypical creaky voice. Oscillogram (top) and fo curve (bottom) of the beginning of a creaky part during a word. The typical creaky part (to the right) is characterized by low f0 (down to $66 \mathrm{~Hz}$ ) and strongly damped and somewhat irregular periods. b FFT spectrum of prototypical creaky voice, the same sample as seen in $\mathbf{a}$, when the voice has turned to creaky; fo $=94 \mathrm{~Hz}$ as shown by the cursor. c FFT spectrum of the same sample as in $\mathbf{a}$, before the voice quality turns into creaky, f0 $=288 \mathrm{~Hz}$.

Fig. 3. a Multiply (double in this case) pulsed creaky voice. The voice does not sound lowpitched although the result of the f0 analysis suggests that (fO $=106 \mathrm{~Hz}$ ). $\mathbf{b}$ FFT of multiply (double in this case) pulsed creaky voice with two sets of harmonics, $1 \mathrm{f0}=106$ $\mathrm{Hz}$ (subharmonic), shown by the cursor, and $2 \mathrm{fO}=212 \mathrm{~Hz}$. The same sound sample as in a. 
Table 1. Prevalence of perceived creakiness and strain in the text reading samples from 104 female speakers

\begin{tabular}{lll}
\hline $\begin{array}{l}\text { Mean of } \\
\text { judgement }\end{array}$ & $\begin{array}{l}\text { Creaky voice, } \\
\%\end{array}$ & $\begin{array}{l}\text { Strainedness, } \\
\%\end{array}$ \\
\hline$<0$ & 22.1 & 02.9 \\
$>0<1$ & 49.0 & 60.6 \\
$>1<2$ & 24.1 & 27.8 \\
$>2<3$ & 04.8 & 08.7 \\
\hline
\end{tabular}

0 , no creakiness/strain; 3, a lot of creakiness/strain. Both qualities were evaluated separately. Percentages refer to the number of speakers who obtained a particular mean value in the evaluation.

Table 2. Prevalence of vocal symptoms in the participants

\begin{tabular}{|c|c|c|c|c|c|}
\hline & $N$ & Min. & Max. & Mean & SD \\
\hline Symptoms total & 051 & 0 & 36 & 005.53 & 0006.52 \\
\hline VHI total & 099 & 1 & 58 & 015.63 & 0010.00 \\
\hline Total F & 100 & 0 & 21 & 004.74 & 3.17064 \\
\hline Total P & 103 & 0 & 20 & 7.9223 & 4.73346 \\
\hline Total E & 104 & 0 & 22 & 2.9231 & 3.66482 \\
\hline $\mathrm{F} 1$ & 100 & 0 & 02 & 000.92 & 0000.73 \\
\hline $\mathrm{F} 2$ & 104 & 0 & 03 & 001.23 & 0000.75 \\
\hline $\mathrm{F} 3$ & 104 & 0 & 03 & 000.84 & 0000.81 \\
\hline $\mathrm{F} 4$ & 104 & 0 & 03 & 000.38 & 0000.64 \\
\hline F5 & 104 & 0 & 02 & 000.15 & 0000.44 \\
\hline F6 & 104 & 0 & 02 & 000.06 & 0000.27 \\
\hline F7 & 104 & 0 & 03 & 000.93 & 0000.77 \\
\hline F8 & 104 & 0 & 02 & 000.14 & 0000.45 \\
\hline F9 & 104 & 0 & 02 & 000.13 & 0000.37 \\
\hline F10 & 104 & 0 & 01 & 000.04 & 0000.19 \\
\hline P1 & 104 & 0 & 03 & 001.29 & 0000.68 \\
\hline P2 & 103 & 0 & 04 & 001.50 & 0000.91 \\
\hline P3 & 104 & 0 & 03 & 000.19 & 0000.56 \\
\hline P4 & 104 & 0 & 03 & 000.88 & 0000.85 \\
\hline P5 & 104 & 0 & 03 & 000.74 & 0000.79 \\
\hline P6 & 104 & 0 & 02 & 000.66 & 0000.76 \\
\hline P7 & 104 & 0 & 02 & 000.53 & 0000.76 \\
\hline P8 & 104 & 0 & 02 & 000.46 & 0000.70 \\
\hline P9 & 104 & 0 & 03 & 000.85 & 0000.90 \\
\hline P10 & 104 & 0 & 02 & 000.78 & 0000.71 \\
\hline E1 & 104 & 0 & 03 & 000.71 & 0000.84 \\
\hline E2 & 104 & 0 & 02 & 000.36 & 0000.64 \\
\hline E3 & 104 & 0 & 03 & 000.20 & 0000.55 \\
\hline E4 & 104 & 0 & 02 & 000.25 & 0000.59 \\
\hline E5 & 104 & 0 & 02 & 000.09 & 0000.34 \\
\hline E6 & 104 & 0 & 03 & 000.17 & 0000.51 \\
\hline E7 & 104 & 0 & 03 & 000.59 & 0000.81 \\
\hline E8 & 104 & 0 & 02 & 000.35 & 0000.60 \\
\hline E9 & 104 & 0 & 03 & 000.12 & 0000.40 \\
\hline E10 & 104 & 0 & 02 & 000.10 & 0000.36 \\
\hline
\end{tabular}

104 Finnish female university students in total answered the Voice Handicap Index (VHI); 51 also filled in a questionnaire about frequency and severity of various symptoms of vocal fatigue. $N$ values for VHI scores vary between 99 and 104, since in total 5 of the participants did not answer all of the VHI statements. VHI subscores are labelled: $\mathrm{F}=$ functional, $\mathrm{P}=$ physical, and $\mathrm{E}=$ emotional (handicap due to voice) [32]. 
Table 3. Correlations between vocal symptom scores and mean perceived creakiness and strain

\begin{tabular}{lll}
\hline Spearman's $\rho$ & Mean creakiness & Mean strain \\
\hline Mean creakiness & & \\
$\quad$ Correlation coefficient & $000 .-1$ & $-0.404^{\star \star}$ \\
Sig. (2-tailed) & $000 .-$ & $000 .-0$ \\
$N$ & $0 .-103$ & $0 .-103$ \\
Mean strain & & \\
$\quad$ Correlation coefficient & $-0.404^{\star \star}$ & $000 .-1$ \\
Sig. (2-tailed) & $000 .-0$ & $000 .-$ \\
$N$ & $0 .-103$ & $0 .-104$ \\
Symptoms total & & \\
Correlation coefficient & -0.035 & -0.206 \\
Sig. (2-tailed) & -0.806 & -0.148 \\
$N$ & $00 .-51$ & $00 .-51$ \\
VHI total & & \\
Correlation coefficient & -0.086 & $0 .-0.04$ \\
Sig. (2-tailed) & -0.401 & -0.694 \\
$N$ & $00 .-98$ & $00 .-99$ \\
Total F & & \\
Correlation coefficient & -0.089 & -0.001 \\
Sig. (2-tailed) & $0 .-0.38$ & -0.989 \\
$N$ & $00 .-99$ & $0 .-100$ \\
Total P & & \\
Correlation coefficient & -0.052 & -0.021 \\
Sig. (2-tailed) & -0.606 & -0.829 \\
$N$ & $0 .-102$ & $0 .-103$ \\
Total E & & -0.038 \\
Correlation coefficient & -0.103 & -0.701 \\
Sig. (2-tailed) & -0.299 & \\
\hline
\end{tabular}

Sig., significance; VHI, Voice Handicap Index. ** Correlation is significant at the 0.01 level (2-tailed).

Table 4. Comparison of vocal symptoms and Voice Handicap Index (VHI) total scores between the groups with more (>1) and less $(<1)$ creakiness or strain (Student's $t$ test, independent samples test)

\begin{tabular}{lllllll}
\hline & Voice quality & $N$ & Mean & SD & SEM & $\begin{array}{l}\text { Sig. } \\
(2 \text {-tailed) }\end{array}$ \\
\hline \multirow{2}{*}{ Symptoms total } & Less creaky & 37 & 05.81 & 06.81 & 1.12 & 0.62 \\
& More creaky & 14 & 04.79 & 05.85 & 1.56 & \\
\hline VHI total & Less creaky & 68 & 16.53 & 10.57 & 1.28 & 0.23 \\
& More creaky & 30 & 13.87 & 08.48 & 1.55 & \\
\hline Symptoms total & Less strained & 30 & 06.43 & 07.32 & 1.34 & 0.24 \\
& More strained & 21 & 04.24 & 05.05 & 1.10 & \\
\hline VHI total & Less strained & 62 & 15.92 & 10.71 & 1.36 & 0.71 \\
& More strained & 37 & 15.14 & 08.81 & 1.45 & \\
\hline
\end{tabular}

Voice quality was perceptually assessed by a speech therapist and a voice trainer on a scale of $0-3 ; 0=$ no creakiness/strain; 3 = a lot of creakiness/strain, and means of the evaluations were calculated. The cut-off point was 1 for the groups. SD, standard deviation; SEM, standard error of the mean; sig., significance. 
fpl506901_f01.jpg

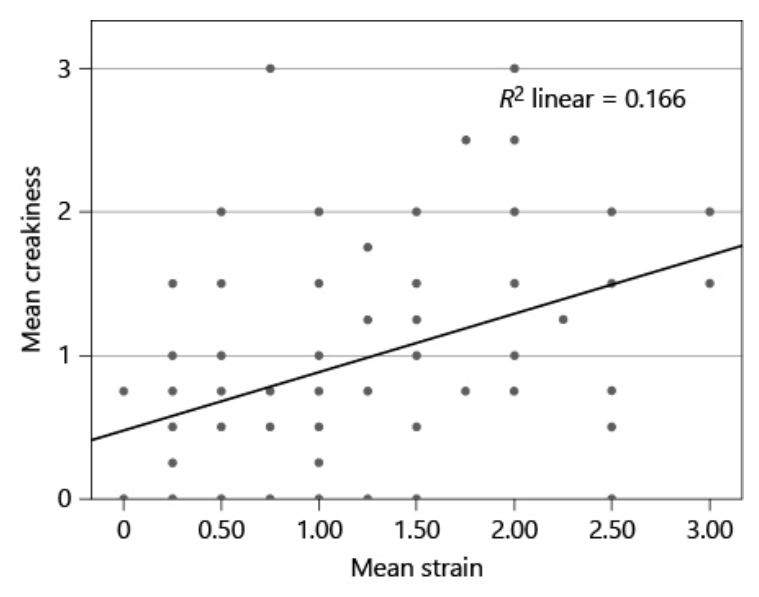


fpl506901_f02.jpg

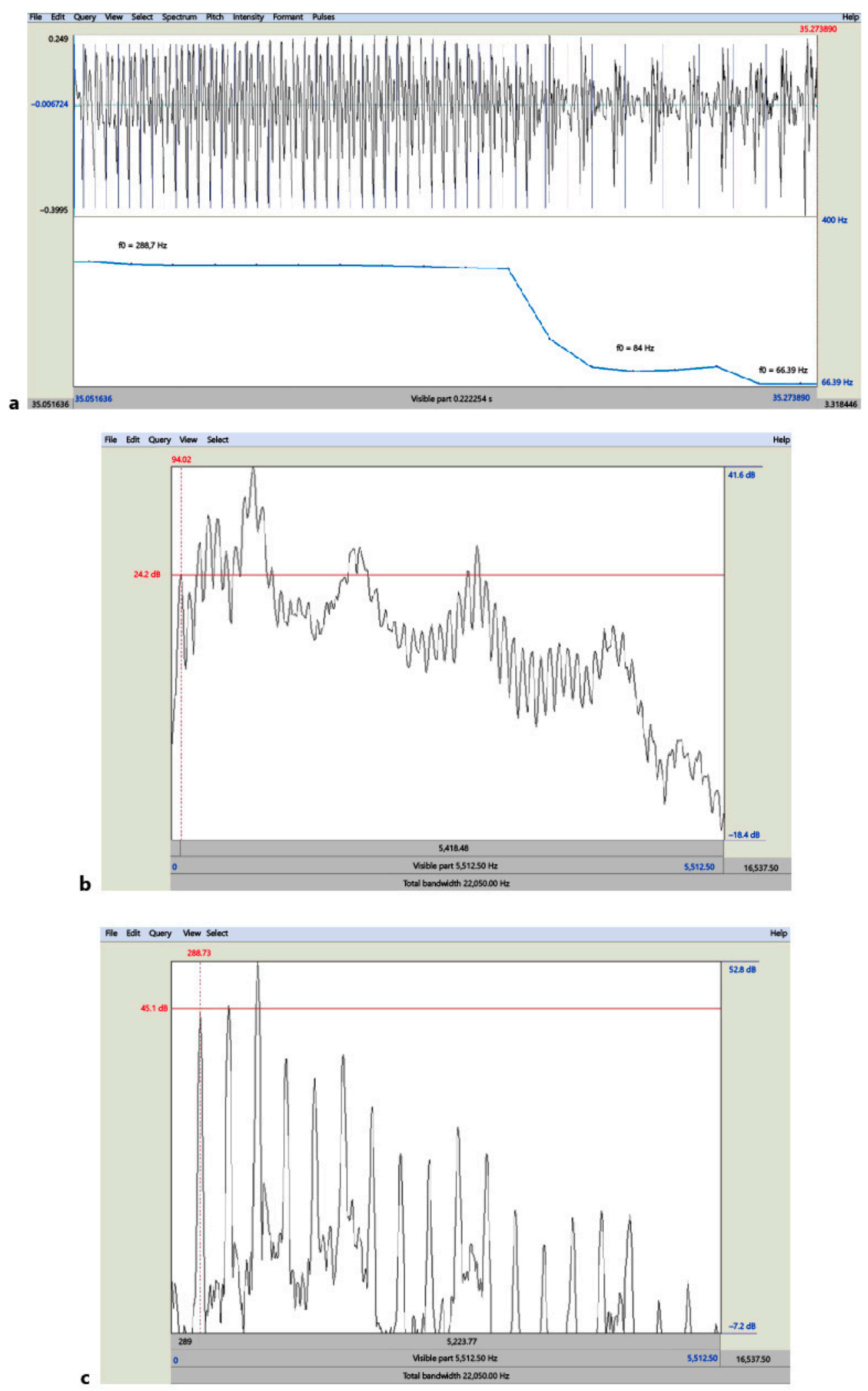


fpl506901_f03.jpg
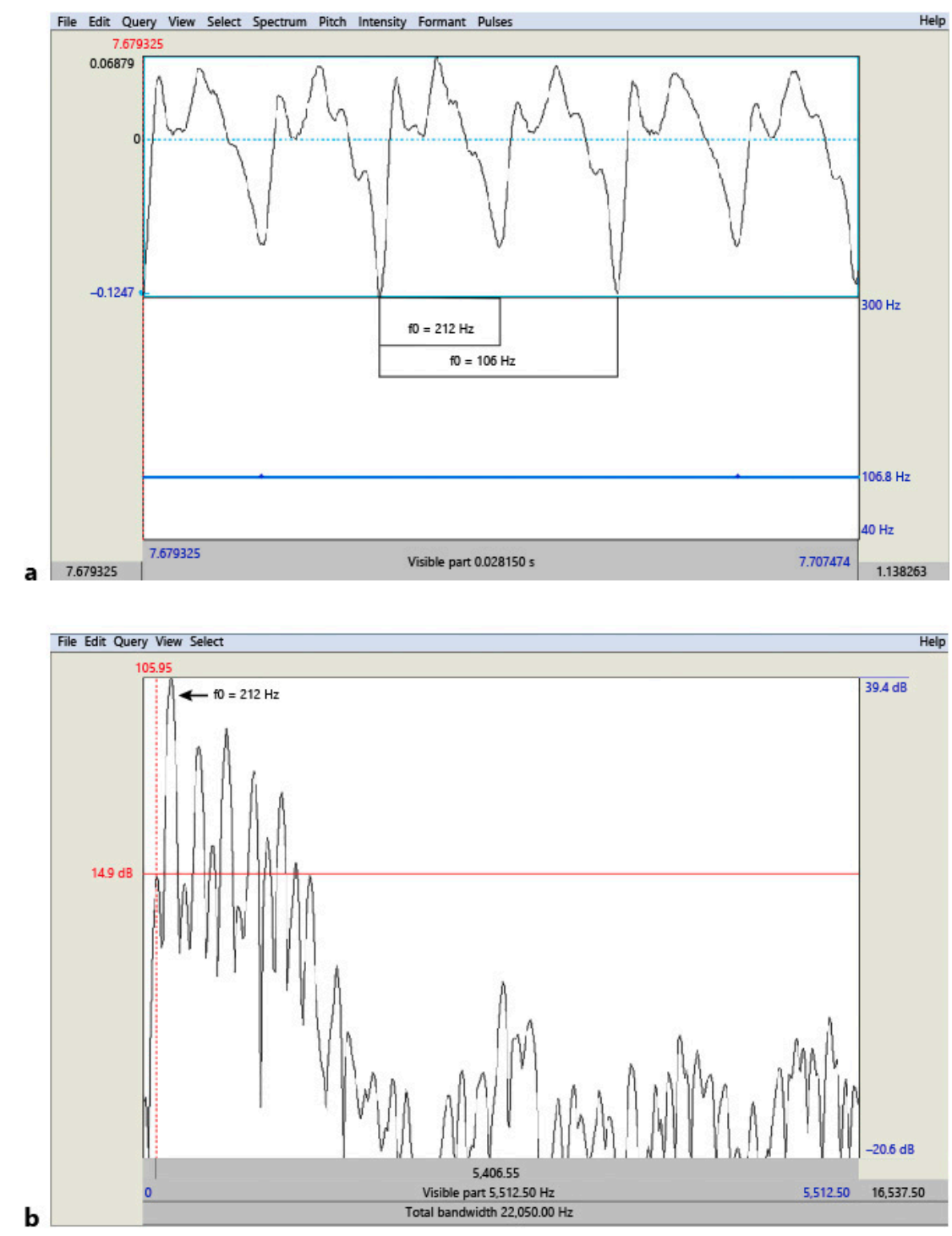